\title{
Single cell atlas of trisomy 21 cerebral cortex
}

Jingxuan Zhang ${ }^{1,2 \#}$, Shiyou Wang ${ }^{3,4 \#}$, Zaoxu Xu ${ }^{3,4 \#}$, Xiangning Ding ${ }^{3,4 \#}$, Chen $\mathrm{Li}^{1 \#}$, Yonghong Zhang ${ }^{1 \#}$, Yin Chen ${ }^{3,4}$, Jixing Zhong ${ }^{3,4,5}$, Langchao Liang, ${ }^{3,4}$ Chaochao Chai $^{3,4}$, Xiaoling Wang ${ }^{3,4}$, Rong Xiang ${ }^{3,4}$, Jiacheng $\mathrm{Zhu}^{3,4}$, Xiumei Lin $^{3,4}$, Peiwen Ding $^{3,4}$, Qiang Zhang ${ }^{3}$, Mingyue Wang ${ }^{3,4}$, Qikai Feng ${ }^{3,4}$, Zhijun Zhang ${ }^{6}$, Guangling $\mathrm{Guo}^{6,7}$, Shen Xue ${ }^{1}$, Lin Jin ${ }^{8}$, Zhikai $\mathrm{He}^{1}$, Li Yan ${ }^{7}$, Bing Xiao ${ }^{8}$, Changjun Zhang ${ }^{9}$, Yan $\mathrm{Xu}^{9}$, Wei $\mathrm{Li}^{3}$, Yichi Zhang ${ }^{3}$, Weiying $\mathrm{Wu}^{3,4}$, Sanjie $\mathrm{Jiang}^{3,4}$, Jun $\mathrm{Xia}^{3,4,10}$, Ya Gao ${ }^{3}$, Lei Wang ${ }^{3,4}$, Shichen Dong ${ }^{3}$, Si Liu ${ }^{3}$, Shida $\mathrm{Zhu}^{3}$, Fang Chen ${ }^{3,10^{*}}$, Dongsheng Chen ${ }^{3 *} \&$ $\mathrm{Xun} \mathrm{Xu}^{3 *}$

1. School of Basic Medical Sciences, Hubei University of Medicine

2. Hubei Key Laboratory of Embryonic Stem Cell Research, Taihe Hospital, Hubei University of Medicine, Shiyan, Hubei 442000, China

3. BGI-shenzhen, Shenzhen 518083, China

4. BGI Education Center, University of Chinese Academy of Sciences, Shenzhen 518083, China

5. School of Biological Science \& Medical Engineering, Southeast University, Nanjing 210096, China

6. Department of gynaecology and obstetrics, Taihe Hospital of Shiyan City

7. Department of Reproductive Medical Center, Taihe Hospital, Hubei University of Medicine , Hubei , China

8. Department of Obstetrics, Shiyan Maternal and Child Health Hospital,Hubei. China

9. Reproductive Medicine Center, Renmin Hospital, Hubei University of Medicine

10. MGI, BGI-Shenzhen, Shenzhen 518083, China

\#These authors contributed equally to this work.

*Correspondence should be addressed to X.X. (xuxun@genomics.cn), D.C. (chendongsheng@genomics.cn), F. C. (fangchen@genomics.cn)

\section{Abstract:}

Down syndrome (DS) is one of the most common human birth defects caused by trisomy 21 (T21), leading to a variety of cognitive impairments. The cellular composition of human brain has been explored using single cell RNA sequencing in both physiological and pathological conditions. 
However, the cellular heterogeneity of human brain with chromosome aneuploidy is largely unknown. Here, we profiled the transcriptome of 36046 cells in cerebral cortex of T21 human fetus, covering frontal lobe, parietal lobe, occipital lobe and temporal lobe. Intriguingly, we detected several genes positively associated with neurons maturation was dysregulated in T21 frontal cortex (HIC2, POU2F2, ZGLP1 and FOXK1). To share, explore and utilized the data resources of T21 cerebral cortex, we developed a comprehensive platform named T21atlas, composing of two functional modules (T21cluster and T21talk). Overall, our study provides, as far as we know, the first single cell atlas for T21 cerebral cortex, which could promote our understanding of the molecular mechanism of DS at an unprecedented resolution and could potentially facilitate the development of novel clinical therapeutics against T21.

\section{Introduction:}

Down syndrome (DS), due to the trisomy of human chromosome 21 (Hsa21), was estimated to occur in the frequency of one in every 700 newborns around the world ${ }^{1}$. Due to the presence of an additional copy of Hsa21 compromising $\sim 250$ genes, DS patients demonstrate a wide spectrum of abnormal phenotypes, including early onset Alzheimer disease, early ageing, congenital cardiac defects and childhood leukemia ${ }^{1}$. T21 leads to the degeneration of the cortical pyramidal neurons, deep 
dendrites, synaptic abnormalities and a reduction of cerebral cortical neurons ${ }^{2}$.

scRNAseq has been widely used to explore the heterogeneity of human cerebral cortex for several years ${ }^{3-6}$. Fan et al. reported the transcriptome of cells from 22 brain regions, resulting in 29 cell sub-clusters with a variety of cells from each region. Zhong et al. obtained the single cell transcriptomes of the developing human pre-frontal cortex ranging from gestation week 8 to 26 , from which they identified six cell types composed of 35 sub-types. Through comprehensive analysis, they revealed the intrinsic developmental signals regulating circuit formation and neuron generation ${ }^{7}$. Additionally, brain cellular compositions under pathological conditions have also been studied using scRNA including $\mathrm{PD}, \mathrm{AD}$ and $\mathrm{ASD}$, revealing cell type and molecular alteration related to pathology $\mathrm{y}^{8-11}$

Encouraged by the potent power of scRNA to dissect neural cell diversity as well as cellular micro-environment and its successful applications to understand the development of neural diseases at high resolution and throughput, we performed scRNAseq for four distinct regions of fetal cerebral cortex at mid-gestation period, giving rise to the transcriptome data of 36046 cells. We studied the cellular heterogeneity using unbiased 
clustering. By comparing our T21 data with previously reported normal brain data ${ }^{7,12}$, we identified dys-regulation of molecular signatures associated with several cell types.

\section{Results}

\section{Generation of $\mathbf{T} 21$ cerebral cortex single cell atlas}

To characterize the molecular features of cells in the cerebral cortex in DS brain, we profiled the transcriptome of 36,046 single cells from frontal cortex (FC), temporal cortex (TC), parietal cortex (PC) and occipital cortex (OC) around gestational weeks 23 (Figure 1a). To classify the major cell types, we performed unsupervised cellular clustering using Seurat ${ }^{13}$ to identify transcriptionally similar cells, resulting in seven cell types: excitatory neurons (EX), inhibitory neurons (IN), neural progenitor cells (NPC), oligodendrocyte precursor cells (OPC), astrocytes (AST), endothelia (EN) and microglia (MG) (Figure 1b).

EX highly express RBFOX1, SATB2 and NEUROD2 (Figure 1c). RBFOX1 regulates transcripts TFs alternative splicing, including several critical neural transcripts ${ }^{14}$ and controls neurons excitation. SATB2 is a TF critical for projection neurons identity and axons function ${ }^{15}$. NEUROD2 is a transcriptional regulator involved in neuronal determination, highly expressed in mature excitatory neurons. IN specifically expressed 
canonical markers such as NXPH1 and GAD2 (Figure 1c). NXPH1 contribute to the configuration of $G A B A$ receptor and synaptic short-term plasticity $^{16}$. GAD2 is a typical marker of mature inhibitory neurons, catalyzing the production of GABA ${ }^{17}$. NPC specifically expressed PAX6, TNC, HOPX, SLC1A3 and GLI3 (Figure 1c). OPC highly expressed $O L I G 1$, promoting the formation and maturation of oligodendrocytes ${ }^{18}$ (Figure 1c). AST were indicated by the high expression of $G F A P^{19}$. MG highly expressed PTPRC, encoding CD45 protein associated with immune function (Figure 1c). EN highly expressed typical markers IGFBP7 and COLAA2 ${ }^{20}$. Besides, we performed Gene Ontology (GO) enrichment analysis based on cell type specific genes. As expected, EX specific genes were enriched in GO terms associated with "synapse organization" and "regulation of synaptic plasticity". IN specific genes were enriched in GO terms like "regulation of membrane potential", "chemical synaptic transmission", "postsynaptic". NPC specific genes were enriched in GO terms associated with proliferation and differentiation. EN enriched GO terms were related to "regulation of vasculature development" and "endothelial cell migration" (Figure 1d).

\section{Cellular heterogeneity within $\mathbf{T} 21$ cerebral cortex}

To further explore the molecular signatures of different cerebral regions, we analyzed the data on the basis of its spatial origin. Briefly, 9326, 4552, 
21359 and 899 cells were derived from FC, TC, PC and OC respectively.

Seven major cell types (EX, IN, NPC, OPC, AST, MG and EN), composing of 20 clusters, were profiled from frontal cortex (FC) (Figure 2a). Specifically, excitatory neurons $(\mathrm{EX})$ are composed of 10 clusters which can be grouped into two classes: FC_EX_1, FC_EX_2, FC_EX_3, FC_EX_4, FC_EX_5, FC_EX_6 and FC_EX_7 highly expressing BFOX1 and NEUROD2 and FC_EX_8, FC_EX_9, FC_EX_10 specifically expressing $S A T B 2$ and $C U X 2$ (Figure 2e). Each cluster was discriminated by a subset of unique molecular signatures (Figure 2b). In particular, DSCAM was highly expressed in FC_EX_2 (Figure 2d). Previous research showed DSCAM was involved in human central and peripheral nervous system development, and this gene was a candidate for Down syndrome ${ }^{21}$.

Likewise, FC inhibitory neurons (IN) can be classified into two groups: FC_IN_1, FC_IN_2 expressing $G A D 1$ and $N X P H 1$ and FC_IN_3, FC_IN_4 expressing ERBB4 and $D L X 6-A S 1^{4}$. NPC is consisted of three subtypes: FC_NPC_1 highly expressing PAX6, FC_NPC_2 and FC_NPC_3 highly expressing SLC1A3. FC_AST, FC_MG and FC_EN were composed of one cluster each. Briefly, AST highly expressed GFAP. MG highly expressed PTPRC, and enriched immune associated GO terms 
including "regulation of lymphocyte activation" and "T cell activation". EN highly expressed IGFBP7 and COLAA2 and enriched GO terms related to "epithelial cell migration" and "endothelial cell differentiation" associated terms (Figure 2f).

Temporal cortex (TC) contribute to 4552 cells (21 clusters) containing six neural cell types (IN, EX, NPC, OPC, AST and MG) and three non-neural cells: endothelial cells (EN), blood cells (BL) and immune cells (IM) (Figure S1). Two clusters (C10, C12), highly expressing SATB2 and NEUROD2, were considered to be mature excitatory neurons. Cluster 2 and 3 (highly expressing SATB2) and cluster 13 expressing $S A T B 2$ and ZEB1 were also annotated as excitatory neurons. Cluster 14 and 18 expressing NXPH1 and GAD1. 21359 cells were retrieved from parietal cortex (PC), from which 18 clusters associated with seven main cell types (IN, EX, NPC, AST, MG and EN) were identified. Occipital cortex (OC) data set is consisting of 899 cells which were subsequently classified into nine clusters related to four main cell types (NPC, EX, IN, $\mathrm{EN})$.

Two group of FC neural progenitor cells with distinct differential potentials and molecular characteristics

To dissect developmental trajectory within FC, we constructed 
developmental trajectories across the whole frontal cortex. Three trajectories were identified using Monocle $3^{22-25}$ (Figure 3a), with the first trajectory (trajectory 1, hereafter termed as T1) mainly composed of T1_NPC (mainly derived from NPC_1 and NPC_2), OPC and AST while the other trajectory (trajectory 2, hereafter termed as T2) mostly composing of T2_NPC (mostly come from NPC_3) and EX. The third trajectory were mainly composed of IN. This observation is in consistent with previous model that NPC can be differentiated into three lineages: AST, OLG and neurons ${ }^{26,27}$. In both trajectories, we noticed that NPC were mainly distributed at the root of the developmental path (Figure 3a). We assumed that T1_NPC and T2_NPC may correspond to neural progenitor cells which have the potential to differentiate into OPC/AST lineages and neuron lineage respectively. To reveal the molecular differences between T1_NPC and T2_NPC, we performed differential gene expression analysis between the two NPC groups. We observed that, genes associated with myelination, were enriched in the T1_NPC, such as $Q K I$ which regulates the differentiation of oligodendrocytes and regulates the myelinating cells of the central nervous system (Figure 3b). We found HES6 preferentially expressed in T2_NPC (Figure 3b), it has been shown to promote cell differentiation in cortical neurogenesis by inhibiting Hes 1 transcription activity in mouse. We found several GO terms specific to each NPC subgroup. Briefly, "myelination", "ensheathment of neurons" 
and "axon ensheathment" were specific to T1_NPC while "nuclear division", "organelle fission" and "chromosome segregation" were unique to T2_NPC (Figure 3c).

\section{FC excitatory neuron maturation regulation}

Next, we investigated genes associated with FC excitatory neurons maturation. In consistent with previous study, $Z E B 1$, the marker of immaturity, declined during the process of EX maturation. Additionally, we found NFIA, ZFHX4, MEIS2, ZBTB20 were down regulated along excitatory neuron maturation (Figure 3d). This was consistent with the previously reported expression patterns of those genes in normal FC excitatory neurons ${ }^{7}$. According to Fan et al., ZGLP1, POU2F2, HIC2 and FOXK1 were up regulated along excitatory neurons maturation process. However, they were expressed in trisomy 21 excitatory neurons at very low levels (Figure 3d), suggesting that the maturation procedure of T21 FC excitatory neurons was distinct from that of EX under physiological condition.

In addition to above mentioned well-characterized neuron maturation regulators, we also identified several putative novel maturation markers (RORB, TSHZ3 and SOX5) in DS excitatory neurons. RORB (regulating barrel formation ${ }^{28}$ ) and $T S H Z 3$ (playing essential roles in cortical 
projection neurons development ${ }^{29}$ ) were up-regulated along FC excitatory neuron maturation trajectory. These up-regulated TFs might be new excitatory neuron maturation markers of DS frontal cortex. In contrast, SOX5, was down regulated along excitatory neuron maturation process, implying its role in inhibiting neuron maturation (Figure S3b).

\section{Cellular communication network among cell subpopulations within cerebral cortex}

Cell clusters were reported to express a distinct set of ligands and receptors, which lays the foundation for cell-cell communications. Here, we predicted the putative interactions within FC clusters based on cluster specific ligands/receptors and known ligand-receptor interactions (Figure 4a). Briefly, neural lineage clusters specific ligands range from 10 in cluster EX_1 to 31 in cluster EX_6, and neural lineage clusters specific receptors range from to 17 in cluster EX_1 to 63 in cluster NPC_2. Communications happened most frequently in EX_6, IN_3 and NPC_2, suggesting that several subtypes of neural lineage cells have more frequent interactions than other cell types. In contrast, only few interactions were observed in EX_1, suggesting that cells were rather isolated compared to other neural cell clusters (Figure 4b). Within neural progenitor cells, we further identified 24 interactions were shared by all clusters while cluster NPC_2 was specific to other clusters (Figure 4a). 
Of particular interest, several interactions have been reported to be important players of neuron function (table s11). For example, EFNB2 and EPHA4, (specifically expressed in EX_2), were proposed that EPHA4 and its EFN binding pairs are expressed by subtype of neuroblasts suggesting the involvement of heterotypic cellular communications and EphA4 signaling functions in NPC niche organization and neuroblast migration in the forebrain in mice ${ }^{30}$. Besides, our study suggests ligands/receptors were expressed in a cluster specific manner (Figure 4e), SEMA3A and its binding partner Plexins such as PLXNA1, PLXNA2 and PLXNA4 exclusively were identified in NPC_2, which could be used to infer the identity of cell clusters.

\section{Genetic regulatory networks of major cell groups in cerebral cortex}

Given that the brain is the most complicated important organ composed of diverse cell types governing by sophisticated genetic regulatory modules. The identification of genetic regulatory networks (GRNs) of brain cell populations might provide us some clues about how TFs adapt to specific cellular environment and regulate downstream targets to execute specific biological functions. Here, we constructed the regulatory networks of excitatory neurons, by using cell type-specific TFs and its predicted target genes (Figure 5b). Briefly, we combined cluster specific TFs of each cell type and considered those TFs as cell type specific 
regulators (Figure 5b).

In the EX_2 cell group, six TFs (MEIS2, BCL11A, SATB2, CUX2, NCOA2 and RFX3) were identified as cell type specific TFs. MEIS2 was regulator of neuron projection guidance and neuron migration ${ }^{31}$. The GO term of MEIS2 target genes enriched in axon development, axon guidance and regulation of synapse organization, etc. (Figure 5d). And the TF motif verified targets of MEIS2 include DSCAM which was specifically expressed in EX_2 and was found to be over-expressed in developing $\mathrm{CNS}^{21}$.

In the EX_5 cell group, five TFs $(M E F 2 C, S O X 5, S A T B 2, X E B 2$ and $B A C H 2$ ) were identified as putative regulators (Figure 5b). Enriched GO terms are mainly related to ion transmembrane transport, cell communication by electrical coupling and modulation of chemical synaptic transmission. Transcriptome analyses revealed MEF2C as putative key regulators in human ASD brains which associated with various genes affected in autism and was a potential therapeutic target for ASD. The MEF2C motif verified target gene (Figure 5e) ANK3 functions in excessive circuit sensitivity and severe epilepsy by regulating the propagation of action potentials through the assemble of sodium gated ion channels, and found expressed in DS patients ${ }^{32}$. 
In the EX_9 group, six TFs (ZFPM2, TSHZ3, FOXP2, SUB1, TOX and SOX5) were identified as cell type specific TFs (Figure 5c). The enriched GO terms suggest a strong connection of secretory protein trafficking. For instance, regulome of FOXP2 converged to specific GO terms including "translational initiation" and "SRP-dependent co-translational protein targeting to membrane". Additionally, FOXP2, by putative interactions with GRM5, CALM3, NRG3 etc., also seems to modulate retrograde synaptic signaling, an essential process in the generation, maturation and plasticity of synaptic junctions. Taken together, we suspected that EX_9 may be in a state that actively synthesizing and releasing neurotransmitters.

\section{T21atlas for the sharing and exploring of T21 cerebral cortex single cell atlas}

In order to enhance the availability of our data resources, we presented an online interface T21atlas, which is composed of mainly two functional modules: T21cluster module showing the clustering results and corresponding markers, T21 talk module covering the putative interaction networks within distinct cell cluster.

\section{Discussion}


Down syndrome has drawn much attentions from researchers in the field of neuroscience. Cognitive impairment is one of the most typical phenotype of DS patient. Although previous studies have investigated DS brain in bulk level, but such data failed to reveal the intrinsic heterogeneity with brain region. Considering the complexity and brain organ, it is necessary to dissect DS in a higher resolution. In this study, we constructed the first single cell atlas of T21 fetal cerebral cortex consisting of 36,046 single cells derived from frontal cortex, parietal cortex, temporal cortex and occipital cortex, revealing the cellular composition and cell population specific molecular signatures.

Neuron maturation is under deliberate regulation by a repertoire of molecules during cortex development. Notably, we found different neural cell distribution in $\mathrm{T} 21 \mathrm{FC}$ and abnormal TFs expression variation in neurons' maturation. Through trajectory analysis, we identified known neuron maturation markers showing consistent (ZEB1, NFIA, ZFHX4, MEIS2, ZBTB2O) and altered (HIC2, POU2F2, ZGLP1 and FOXK1) expression patterns in T21 FC. HIC2 is a key TF encoding gene involved in cytokine-cytokine receptor interaction ${ }^{38}$. ZGLP1 is a repressor of GATA family playing important roles in regulating cell growth ${ }^{39}$. FOXK1 is reported as the key regulator of neuron maturation ${ }^{7}$. POU2F2 is a bifunctional regulator repressed or induced neuron differentiation. These 
dys-regulated TFs might be related to abnormalities of neurons and CNS. Also, we detected several neural markers (RORB and TSHZ3) which has not been characterized before. We assumed that these up-regulated TFs might represent new excitatory neuron maturation markers controlling FC neuron development.

A distinctive set of TFs were identified from each cell type, representing master regulators coordinating the expression patterns of hundreds downstream targets. To dissect the genetic regulatory network of those cell type TFs, we predicted the targets of each TF based on co-expression patterns. The proliferation, lineage commitment, maturation, reprogramming and apoptosis of a certain cell type is largely determined by several TFs and their genetic regulatory network. Thus, dissecting the regulatory circuits is of crucial importance to understand organism and tissue development. Here, we inferred the targets of TFs based on the Pearson correlation co-efficiency of $\mathrm{TF}$ against genes based on the rationale that genes within the same expression module tend to be co-regulated. As each cell types are composed of hundreds to thousands of cells. The genes co-varying at such a sample sale would be very likely to be correlated. And the correlation at single cell level are expecting to reveal TF-target interaction at a sensitive and high-resolution manner. As expected, we predicted a significant proportion of targets for each $\mathrm{TF}$. 
Among those putative targets, some are well known neural developmental genes.

The single cell resolution defined the characteristics of Down syndrome in a more accurate and sensitive manner compared to traditional methods such as pathology, histology, cell type maker staining and bulk transcriptome, genomic or epigenetic study. Cells are the basic unit of biological functions, exploring the composition of DS brain is of fundamental importance for understanding any degenerative disease. Thus, our data could hopefully serve as the taxonomy of DS cerebral cortex and lays the foundation for future study of DS our findings revealed new insights for investigating the mechanism behind neurologic disease and exploring potential therapies.

\section{Material and methods}

\section{Ethical statement}

Samples were collected with the approval of Medical Ethics Committee of Shiyan Taihe Hospital (201813) following the Code of Ethics of the World Medical Association.

\section{DS brain collection and dissection}

Down syndrome samples collection and dissection was performed the 
same as previously described ${ }^{40}$. Cerebral cortex dissected from $23 \mathrm{GW}$ fetal brain were separated into small pieces and quick frozen in liquid nitrogen and stored in -80 freezer.

\section{Single cell RNA sequencing library preparation and sequencing}

Nuclei were extracted from frozen brain tissues following previous method. Single nuclei RNA sequencing libraries were constructed using 10X Genomics kit (Chromium ${ }^{\mathrm{TM}}$ Single Cell 3'v2) following manufacturer's instruction. Details of library preparation and sequencing were consistent with methods previously reported ${ }^{40}$.

\section{Processing of raw single-nucleus RNA-seq data}

Raw sequencing data was processed by Cell Ranger 3.0.2 (10X Genomics). Then, Seurat 2.3.4 $4^{41}$ was applied for downstream analysis. Furthermore, we filtered the data using following criteria: (1) Genes with the UMI under 3 were discarded; (2) Cells with mitochondrial genes percentage greater than $10 \%$ were filtered out; (3) Cells with genes number more than 2500 or less than 200 were discarded.

\section{Cell clustering}

Seurat package (v2.3.4) were applied to identify major cell types. Briefly, we used the function FindVariableGenes in Seurat to select the highly 
variable genes (HVGs) based on the normalized expression matrix (log (UMI/sum (UMI)*10000)). HVGs whose average expression greater than 0.5 and dispersion between 0.0125 and 3 were used as inputs for the downstream analysis. Then we used the function FindClusters in Seurat, to identify cell clusters based on HVGs (for details, see http://satijalab.org/seurat/pbmc-tutorial.html).

\section{Cluster specific gene identification and GO enrichment analysis}

The function FindAllMarkers built-in Seurat were used to identify cluster-specific marker genes (thresh.use $=0.25$, min.pct $=0.25$, only.pos $=$ TRUE). $\mathrm{R}$ package clusterProfiler (10.1089/omi.2011.0118) were employed for the GO term enrichment of cluster specific genes and $\mathrm{BH}$ method was used for multiple test correction. GO terms and KEGG terms with $\mathrm{P}$ values less than 0.05 were considered as significantly enriched. As for DSCAM, Student t test analysis of its expression levels between excitatory neurons and other clusters was performed. In addition, expression levels between cluster $1(\mathrm{EX})$ and other EX clusters was examined using t test.

\section{Developmental trajectory re-construction}

Monocle ${ }^{42}$ was used for developmental trajectory construction based on the set of variable genes, which were selected using the Seurat R package 
41, with default parameters except we specified reduction_method = "DDRTree" in the reduce dimension function.

\section{TFs target gene prediction}

GENIE3 ${ }^{43}$ was used to predict putative target genes of each TF. Then significant target genes for network construction by cytoscape ${ }^{56}$. And targets with transcription factor motifs present in candidate promoter regions were highlighted. Cytoscape $3 \cdot 6.0^{44}$ was used to visualize the regulation.

\section{Cellular communication network}

Receptor-ligand relationships were downloaded from IUPHAR/BPS Guide to PHARMACOLOGY ${ }^{45}$ and Database of Ligand-Receptor Partners $(\mathrm{DLRP}))^{40}$. "UMI=0.15" was set as the threshold. Cytoscape was used for the visualization of interactions.

\section{Data and software availability}

This data reported in this study are available in the CNGB Nucleotide Sequence Archive (CNSA: https://db.cngb.org/cnsa; accession number CNP0000816 and CNP0000442)

\section{Funding}


D.C. is supported by China Postdoctoral Science Foundation (grant 2017M622795). This research is funded by the Health Commission of Hubei Province Scientific Research Project (WJ2019M051, ZY2019Q004 ), the Natural Science Foundation of Hubei Provincial Department of Education (Q20182104), the Free Exploration Project of Hubei University of Medicine (FDFR201609), Cultivating Project for Young Scholar at Hubei University of Medicine (2017QDJZR26, 2016QDJZR11, 2016QDJZR14) and Shenzhen Municipal Government of China JCYJ20180703093402288.

\section{Acknowledgements}

We are thankful to the production team of China National Gene Bank, Shenzhen, China. We appreciate the constructive feedbacks from Professor Guoji Guo.

\section{Reference}

1 Antonarakis, S. E. Down syndrome and the complexity of genome dosage imbalance. Nature Reviews Genetics 18, 147 (2017).

2 Haydar, T. F. \& Reeves, R. H. Trisomy 21 and early brain development. Trends in neurosciences 35, 81-91 (2012).

3 Darmanis, S. et al. A survey of human brain transcriptome diversity at the single cell level. Proceedings of the National Academy of 
Sciences 112, 7285-7290 (2015).

4 Lake, B. B. et al. Neuronal subtypes and diversity revealed by single-nucleus RNA sequencing of the human brain. Science 352, 1586-1590 (2016).

5 Nowakowski, T. J. et al. Spatiotemporal gene expression trajectories reveal developmental hierarchies of the human cortex. Science 358, 1318-1323 (2017).

6 Polioudakis, D. et al. A single cell transcriptomic analysis of human neocortical development. bioRxiv, 401885 (2018).

7 Zhong, S. et al. A single-cell RNA-seq survey of the developmental landscape of the human prefrontal cortex. Nature 555, 524 (2018).

8 Tirosh, I. et al. Single-cell RNA-seq supports a developmental hierarchy in human oligodendroglioma. Nature 539, 309 (2016).

9 Lang, C. et al. Single-cell sequencing of iPSC-dopamine neurons reconstructs disease progression and identifies HDAC4 as a regulator of parkinson cell phenotypes. Cell stem cell 24, 93-106. e106 (2019).

10 Mathys, H. et al. Single-cell transcriptomic analysis of Alzheimer's disease. Nature, 1 (2019).

11 Velmeshev, D. et al. Single-cell genomics identifies cell type-specific molecular changes in autism. Science 364, 685-689 (2019). 
12 Fan, X. et al. Spatial transcriptomic survey of human embryonic cerebral cortex by single-cell RNA-seq analysis. Cell research $\mathbf{2 8}$, $730(2018)$.

13 Satija, R., Farrell, J. A., Gennert, D., Schier, A. F. \& Regev, A. Spatial reconstruction of single-cell gene expression data. Nature biotechnology 33, 495 (2015).

14 Kuroyanagi, H. Fox-1 family of RNA-binding proteins. Cellular and Molecular Life Sciences 66, 3895 (2009).

15 Alcamo, E. A. et al. Satb2 regulates callosal projection neuron identity in the developing cerebral cortex. Neuron 57, 364-377 (2008).

16 Born, G. et al. Modulation of synaptic function through the $\alpha$-neurexin-specific ligand neurexophilin-1. Proceedings of the National Academy of Sciences 111, E1274-E1283 (2014).

17 Grone, B. P. \& Maruska, K. P. Three distinct glutamate decarboxylase genes in vertebrates. Scientific reports 6, 30507 (2016).

18 Jakovcevski, I. \& Zecevic, N. Olig transcription factors are expressed in oligodendrocyte and neuronal cells in human fetal CNS. Journal of Neuroscience 25, 10064-10073 (2005).

19 Carvalho, F. et al. Neurons induce GFAP gene promoter of cultured astrocytes from transgenic mice. Glia 26, 97-108 (1999). 
20 Zhao, Q. et al. Single-cell transcriptome analyses reveal endothelial cell heterogeneity in tumors and changes following antiangiogenic treatment. Cancer research 78, 2370-2382 (2018).

21 Simmons, A. B. et al. DSCAM-mediated control of dendritic and axonal arbor outgrowth enforces tiling and inhibits synaptic plasticity. Proceedings of the National Academy of Sciences 114, E10224-E10233, doi:10.1073/pnas.1713548114 (2017).

22 Cao, J. et al. The single-cell transcriptional landscape of mammalian organogenesis. Nature 566, 496 (2019).

23 Qiu, X. et al. Reversed graph embedding resolves complex single-cell trajectories. Nature methods 14, 979 (2017).

24 Qiu, X. et al. Single-cell mRNA quantification and differential analysis with Census. Nature methods 14, 309 (2017).

25 Trapnell, C. et al. The dynamics and regulators of cell fate decisions are revealed by pseudotemporal ordering of single cells. Nature biotechnology 32, 381 (2014).

26 Florio, M. \& Huttner, W. B. Neural progenitors, neurogenesis and the evolution of the neocortex. Development 141, 2182-2194 (2014).

$27 \mathrm{Xu}, \mathrm{W} .$, Lakshman, N. \& Morshead, C. M. Building a central nervous system: The neural stem cell lineage revealed. Neurogenesis 4, e1300037 (2017). 
28 Oishi, K. et al. Identity of neocortical layer 4 neurons is specified through correct positioning into the cortex. Elife 5, e10907 (2016).

29 Caubit, X. et al. TSHZ3 deletion causes an autism syndrome and defects in cortical projection neurons. Nature genetics 48, 1359 (2016).

30 Todd, K. L. et al. EphA4 regulates neuroblast and astrocyte organization in a neurogenic niche. Journal of Neuroscience 37, 3331-3341 (2017).

31 Zha, Y. et al. MEIS2 is essential for neuroblastoma cell survival and proliferation by transcriptional control of M-phase progression. Cell death \& disease 5, e1417 (2014).

32 Pathak, A., Agarwal, D. \& Phadke, S. R. Microarray based global transcriptome profiling reveals involvement of non-Hsa21 genes and microRNAs in molecular mechanism of Down syndrome pathogenesis. Molecular cytogenetics 7, P132 (2014).

33 Sheng, L., Leshchyns' ka, I. \& Sytnyk, V. Neural Cell Adhesion Molecule 2 (NCAM2)-Induced c-Src-Dependent Propagation of Submembrane Ca2+ Spikes Along Dendrites Inhibits Synapse Maturation. Cerebral Cortex 29, 1439-1459 (2018).

34 Benhaourech, S. \& Drighil, A. Congenital heart disease and Down syndrome: various aspects of a confirmed association. Cardiovascular journal of Africa 27, 287 (2016). 
35 Marechal, D., Pereira, P. L., Duchon, A. \& Herault, Y. Dosage of the Abcg1-U2af1 region modifies locomotor and cognitive deficits observed in the Tc1 mouse model of Down syndrome. PLoS One 10, e0115302 (2015).

36 Shim, S., Kwan, K. Y., Li, M., Lefebvre, V. \& Šestan, N. Cis-regulatory control of corticospinal system development and evolution. Nature 486, 74 (2012).

37 Mrak, R. E. \& Griffin, W. S. T. Trisomy 21 and the brain. Journal of Neuropathology \& Experimental Neurology 63, 679-685 (2004).

38 Turner, M. D., Nedjai, B., Hurst, T. \& Pennington, D. J. Cytokines and chemokines: at the crossroads of cell signalling and inflammatory disease. Biochimica et Biophysica Acta (BBA)-Molecular Cell Research 1843, 2563-2582 (2014).

39 Lentjes, M. H. et al. The emerging role of GATA transcription factors in development and disease. Expert reviews in molecular medicine 18 (2016).

40 Wu, J. et al. Single cell RNA sequencing reveals cellular diversity of trisomy 21 retina. bioRxiv, 614149 (2019).

41 Maaten, L. v. d. \& Hinton, G. Visualizing data using t-SNE. Journal of machine learning research 9, 2579-2605 (2008).

42 Trapnell, C. et al. Pseudo-temporal ordering of individual cells reveals dynamics and regulators of cell fate decisions. Nature 
biotechnology 32, 381 (2014).

43 Irrthum, A., Wehenkel, L. \& Geurts, P. Inferring regulatory networks from expression data using tree-based methods. PLoS One 5, e12776 (2010).

44 Gu, Z., Gu, L., Eils, R., Schlesner, M. \& Brors, B. circlize implements and enhances circular visualization in $R$. Bioinformatics 30, 2811-2812 (2014).

45 Ravishankar, H. et al. Characterization of NCC-RbC-51, an RB cell line isolated from a metastatic site. Histochemistry and cell biology, 1-9 (2019). 
Figure 1

a

bioRxiv preprint doi: https://doi.org/10.1101/2020.01.01.892398; this version posted January 2, 2020. The copyright holder for this preprint (which was not certifieg by peer review) is the author/funder. All rights reserved. No reuse allowed without permission.

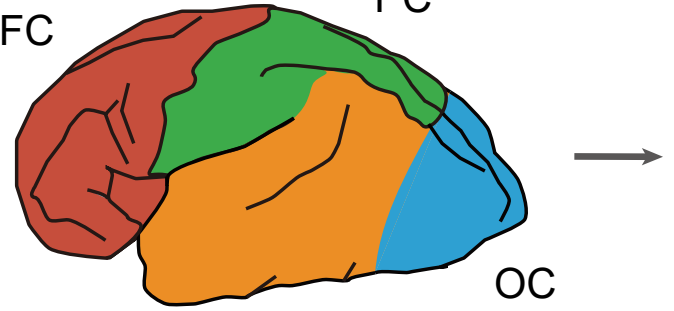

TC
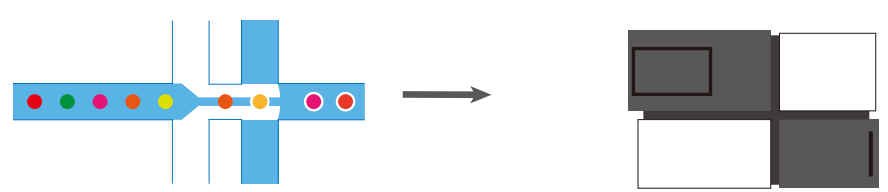

b

C

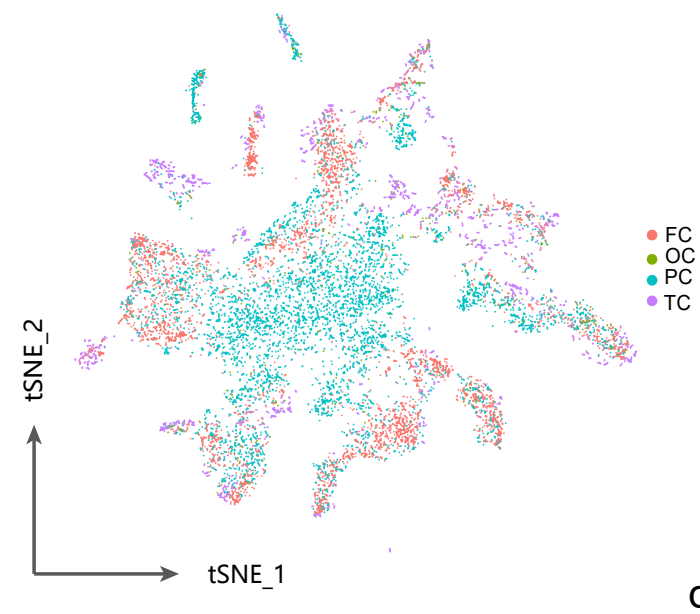

ATP13A4

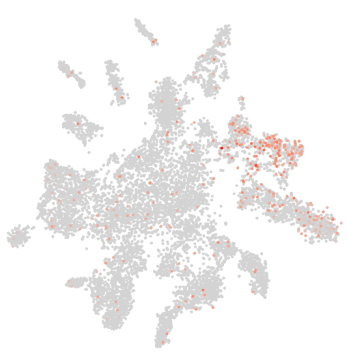

PTPRC

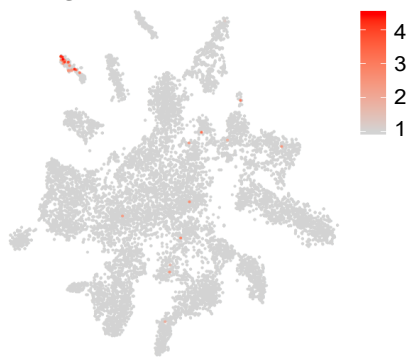

GAD2

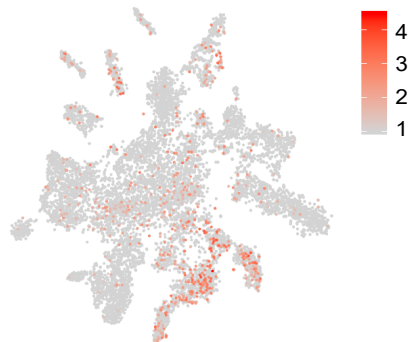

NEUROD2

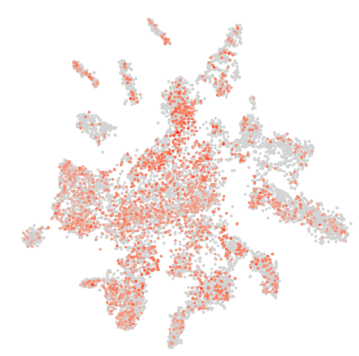

PAX6

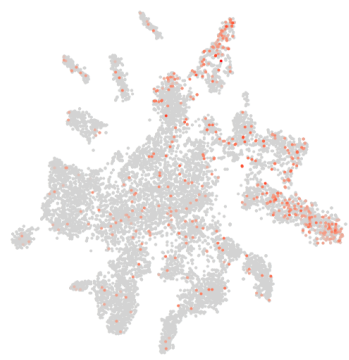

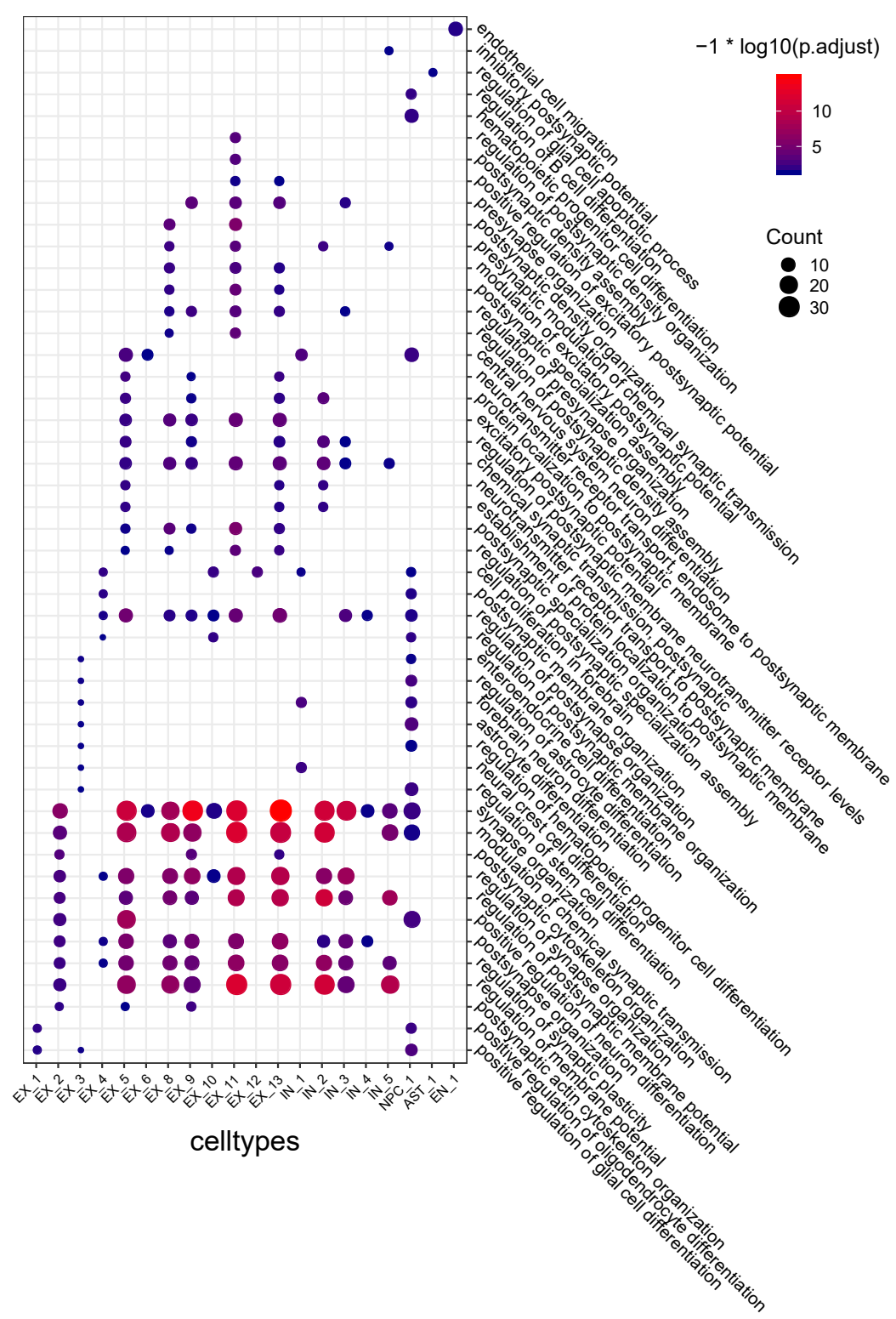

OLIG1

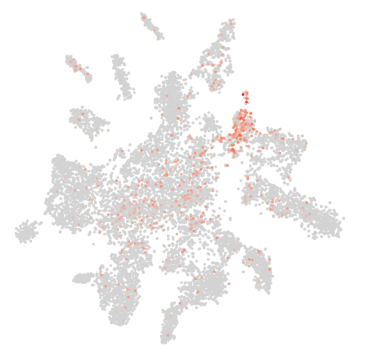

d

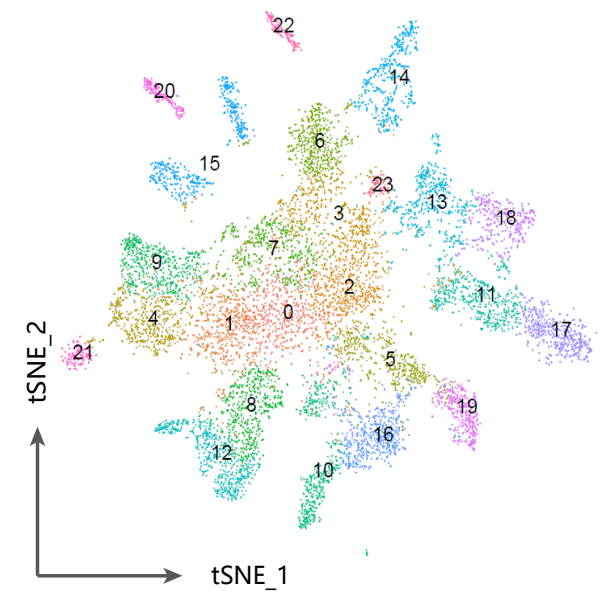

o EX_1

(1) Ex-2

(2) EX_3

4 EX 5

(5) IN_1

6 EX_6
(7) EX-7

$8 \mathrm{EX}^{2} 8$

9 EX_9

(1) IN_2

$12 \mathrm{EX}_{1} 11$

(13) $\mathrm{EPC}_{-12}$

(14) EX_12

16 IN_4

17 NPC_1

19 IN_5

$20 \mathrm{MG}_{1} 1$

(2)

22 EN_1
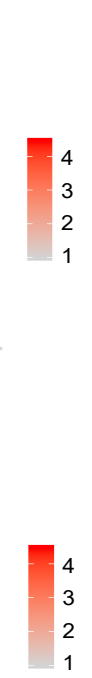

1

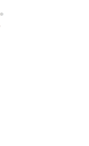

4
3
2
1 
Figure 2

a

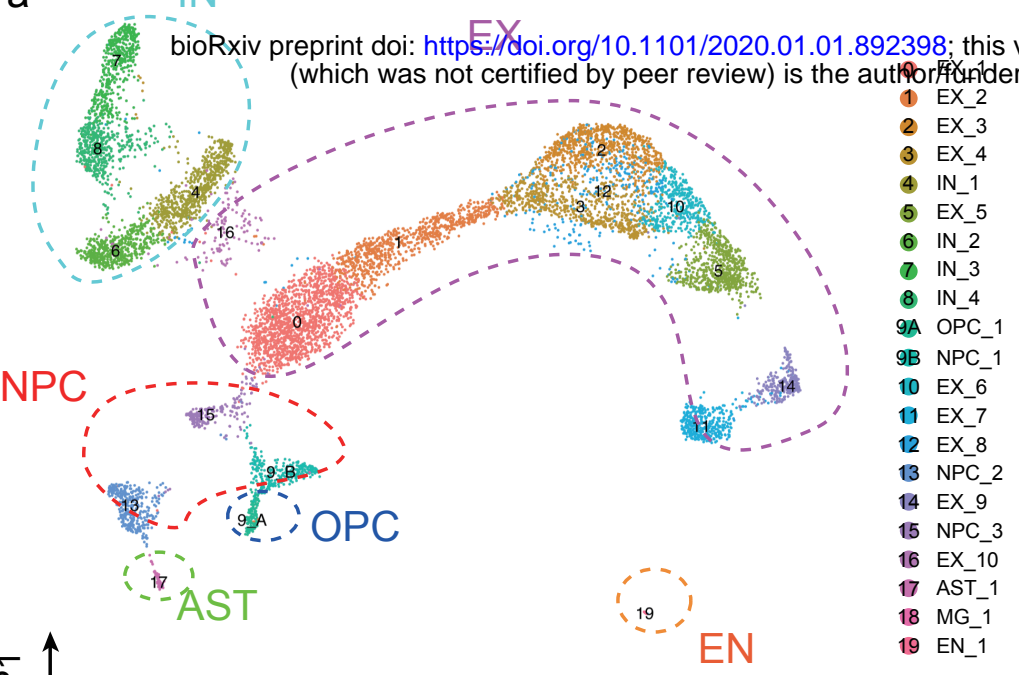

b

$\begin{array}{lllll}-2 & -1 & 0 & 1 & 2\end{array}$

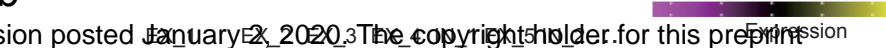

All rights reserved. No reuse allowed without permission.

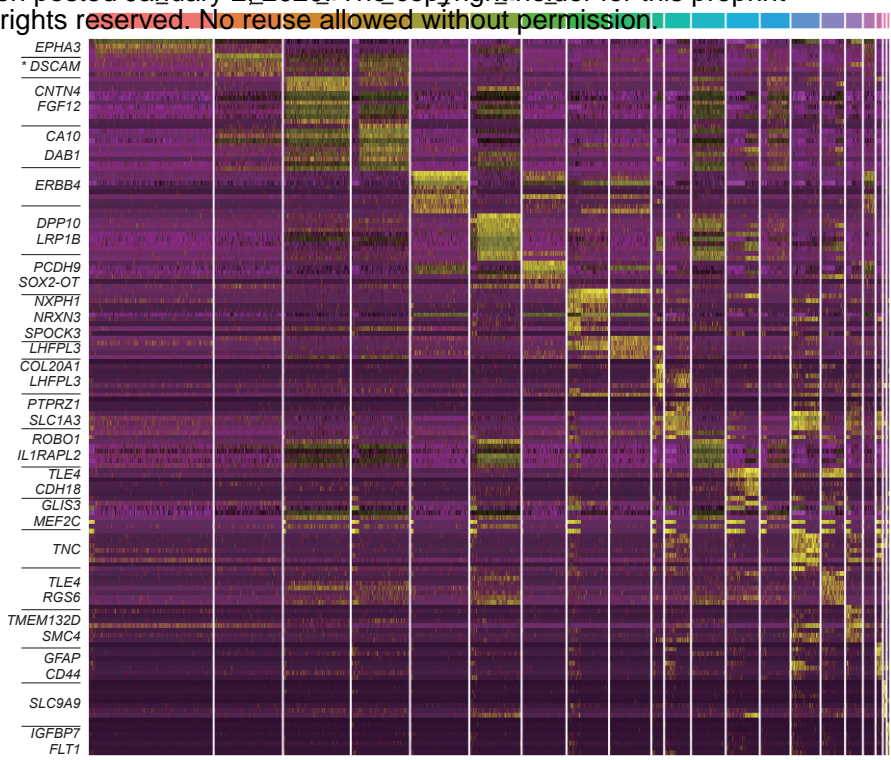

\section{DSCAM}

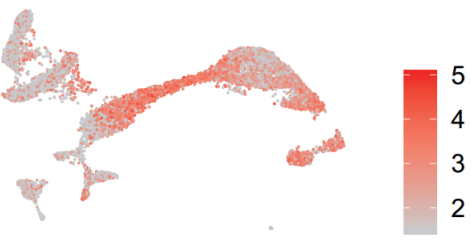

d
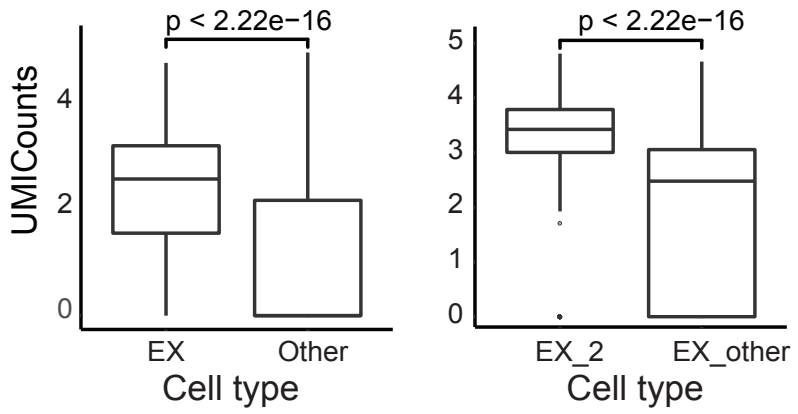

e

Size Freq

- 500

- 1500

AST

$A Q P 4$

OPC

MG

NPC

EX

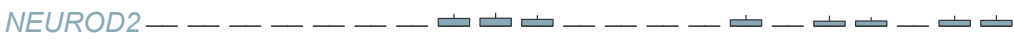

$\operatorname{RORB}-\ldots-\ldots-\ldots-\ldots-\ldots-\ldots$

SATB2

IN

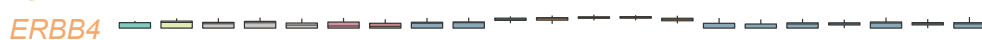

EN

COL4A2

IGFBP7

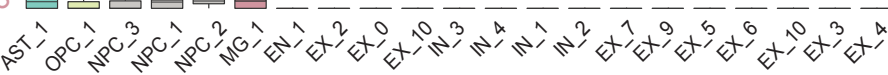

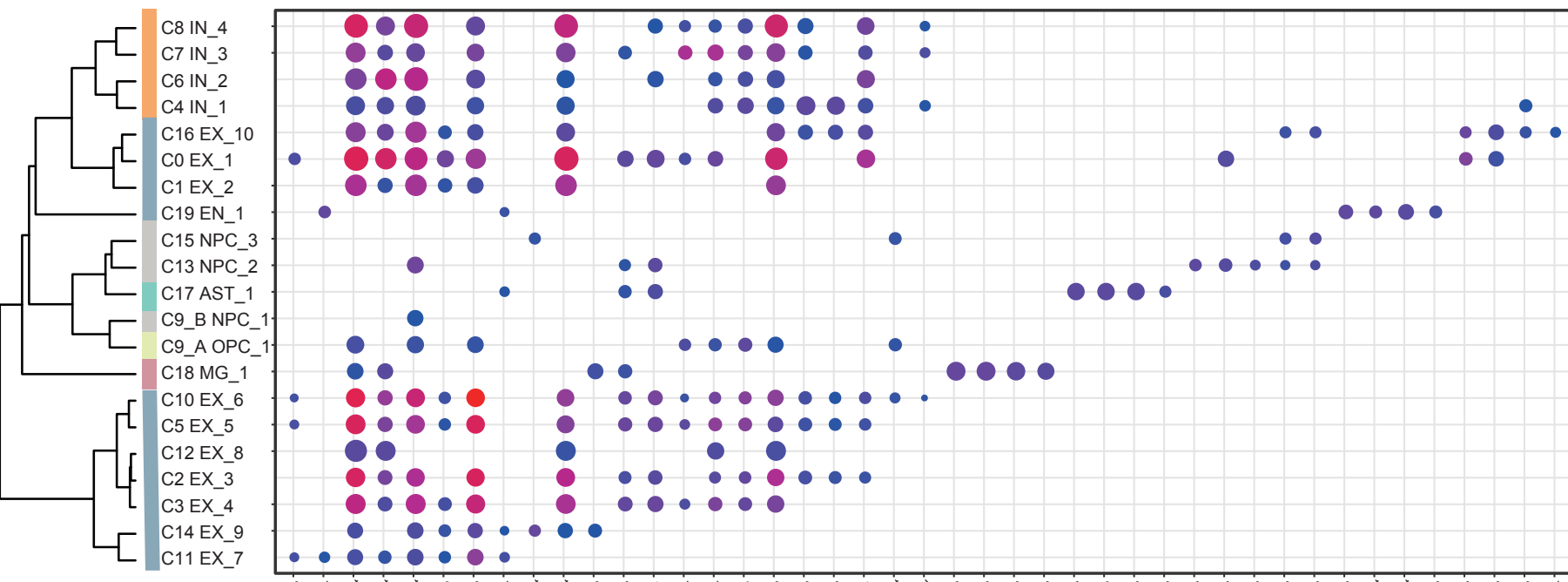

GeneRatio

- 0.05

0.10
0.15

* $\log 10$ ( p.adjust)

-10
-5 


\section{Figure 4}

bioRxiv preprint doi: https://doi.org/10.1101/2020.01.01.892398; this version posted January 2 2020. The copyright holder for this preprint (whiclexaponot Ertgied by peer review) is the author/funder. All rights reserved. No reuse Ellowed without permission.
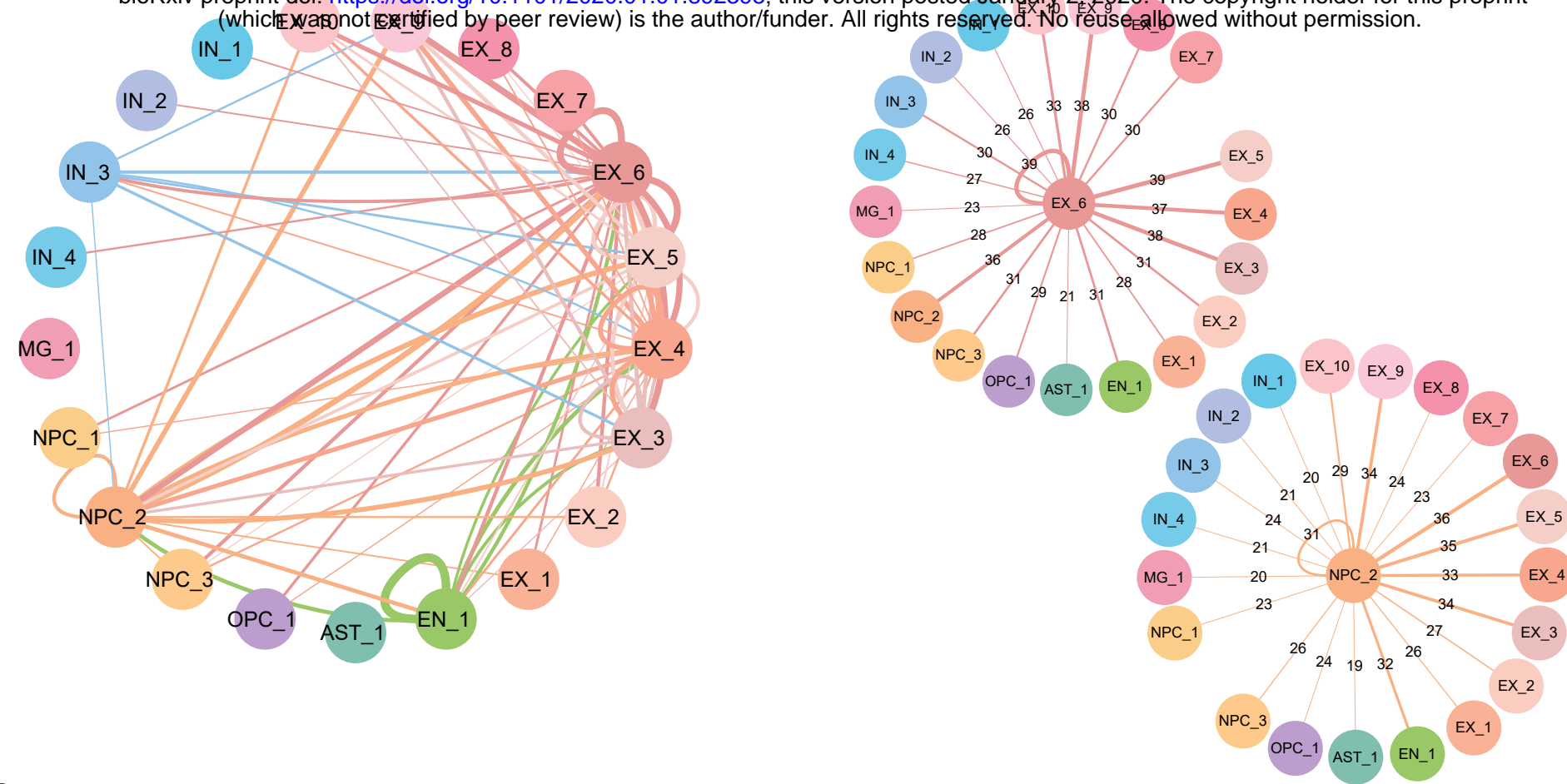

C
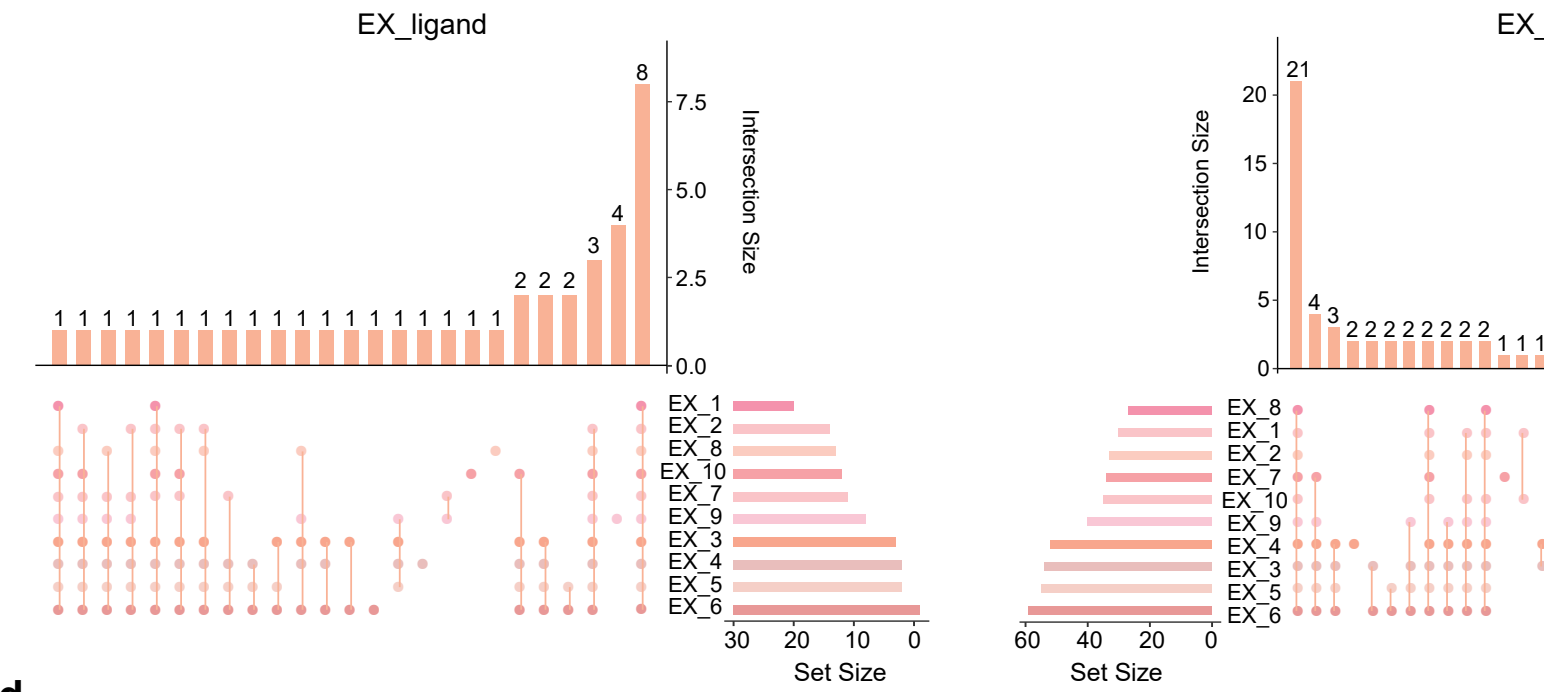

EX_receptor

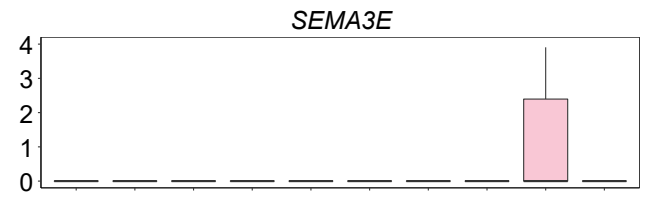

IL7

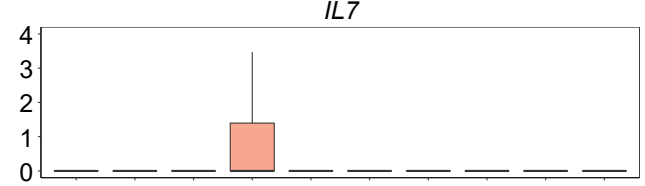

ACVR1B

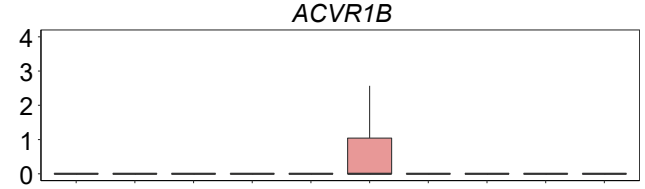

$\mathrm{ROBO} 2$

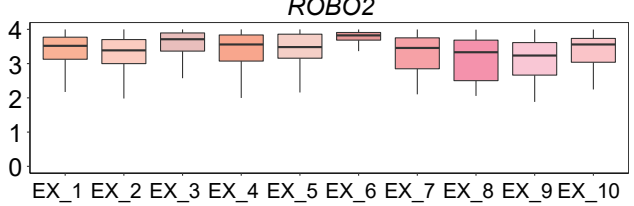

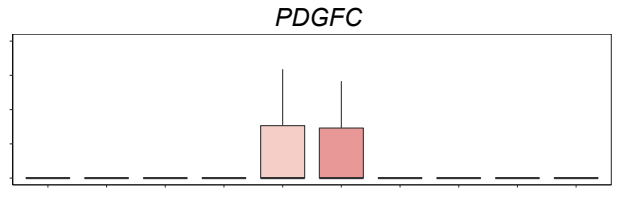

$A P P$

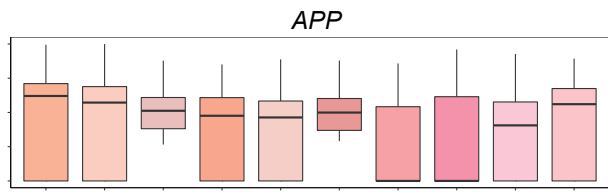

FGFR2

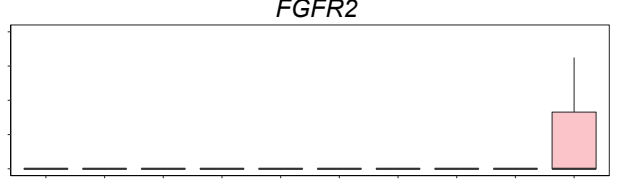

CD47

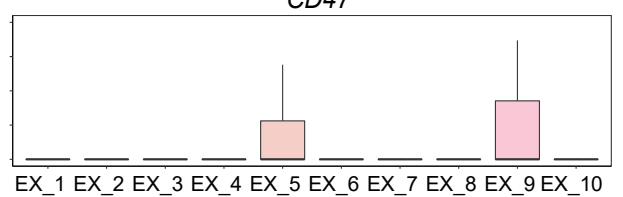

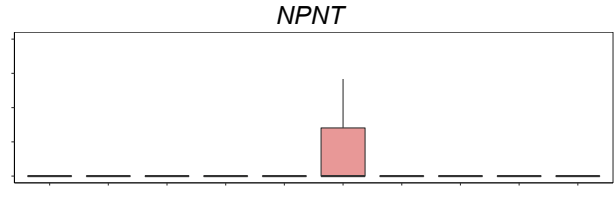

NRG2

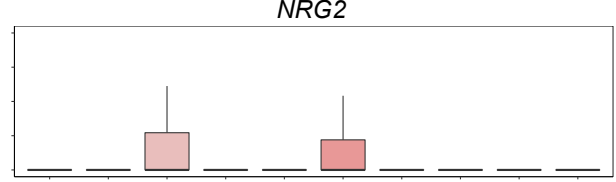

LRP8

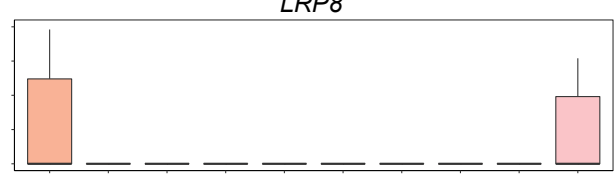

UNC5C

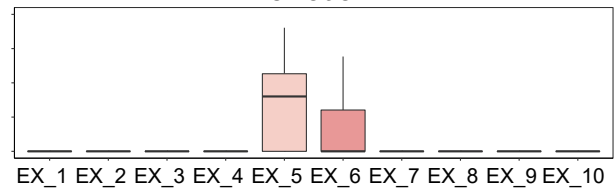


bioRxiv preprint doi: https://doi.org/10.1101/2020.01.01.892398; this version posted January 2, 2020. The copyright holder for this preprint

Figure 5 (which was not certified by peer review) is the author/funder. All rights reserved. No reuse allowed without permission.

a

\section{b}

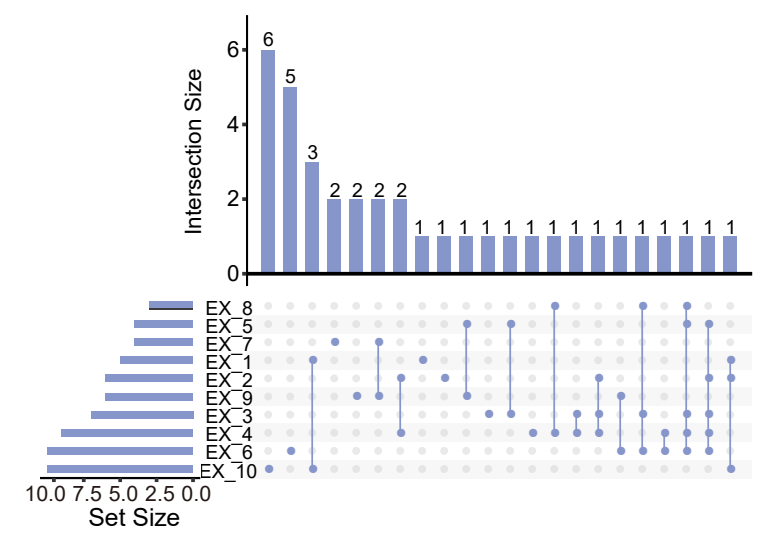

\section{EX_2}

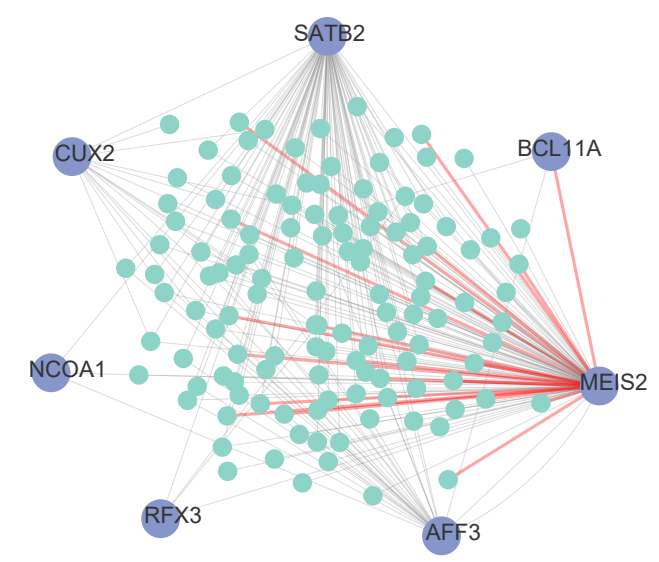

EX-5

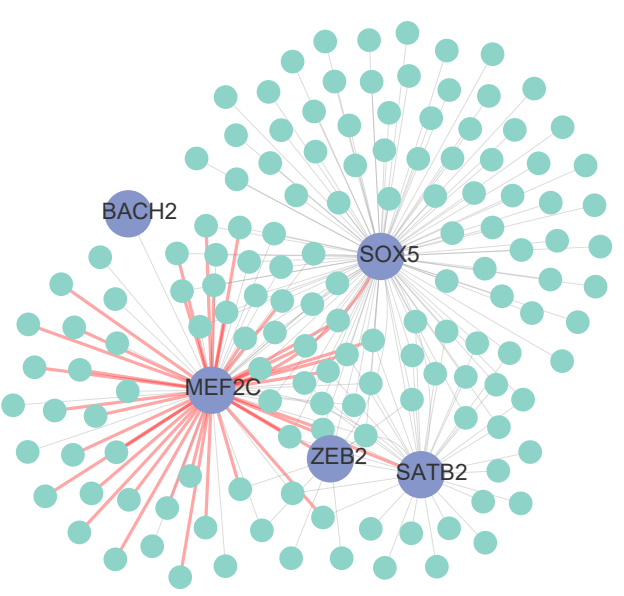

EX_9

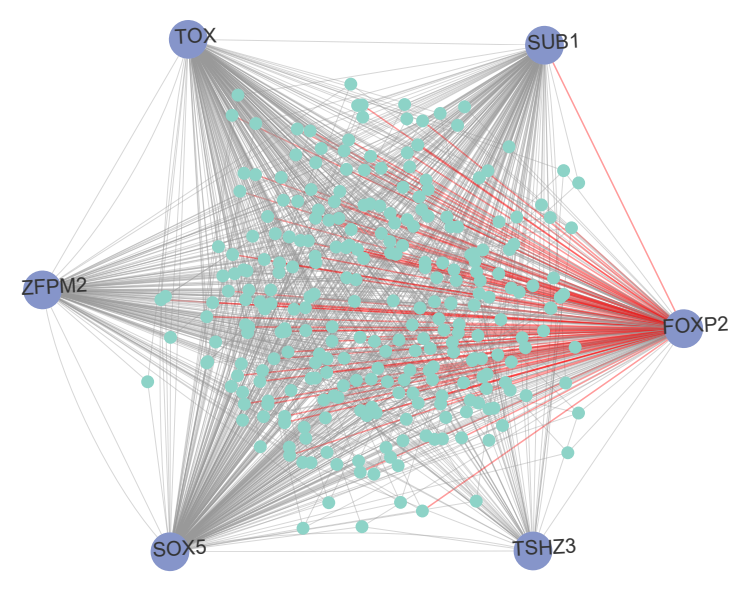

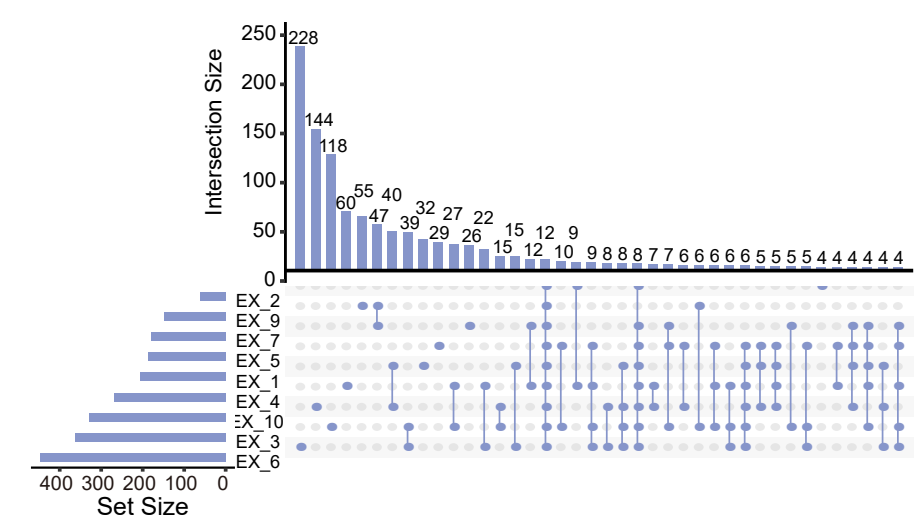
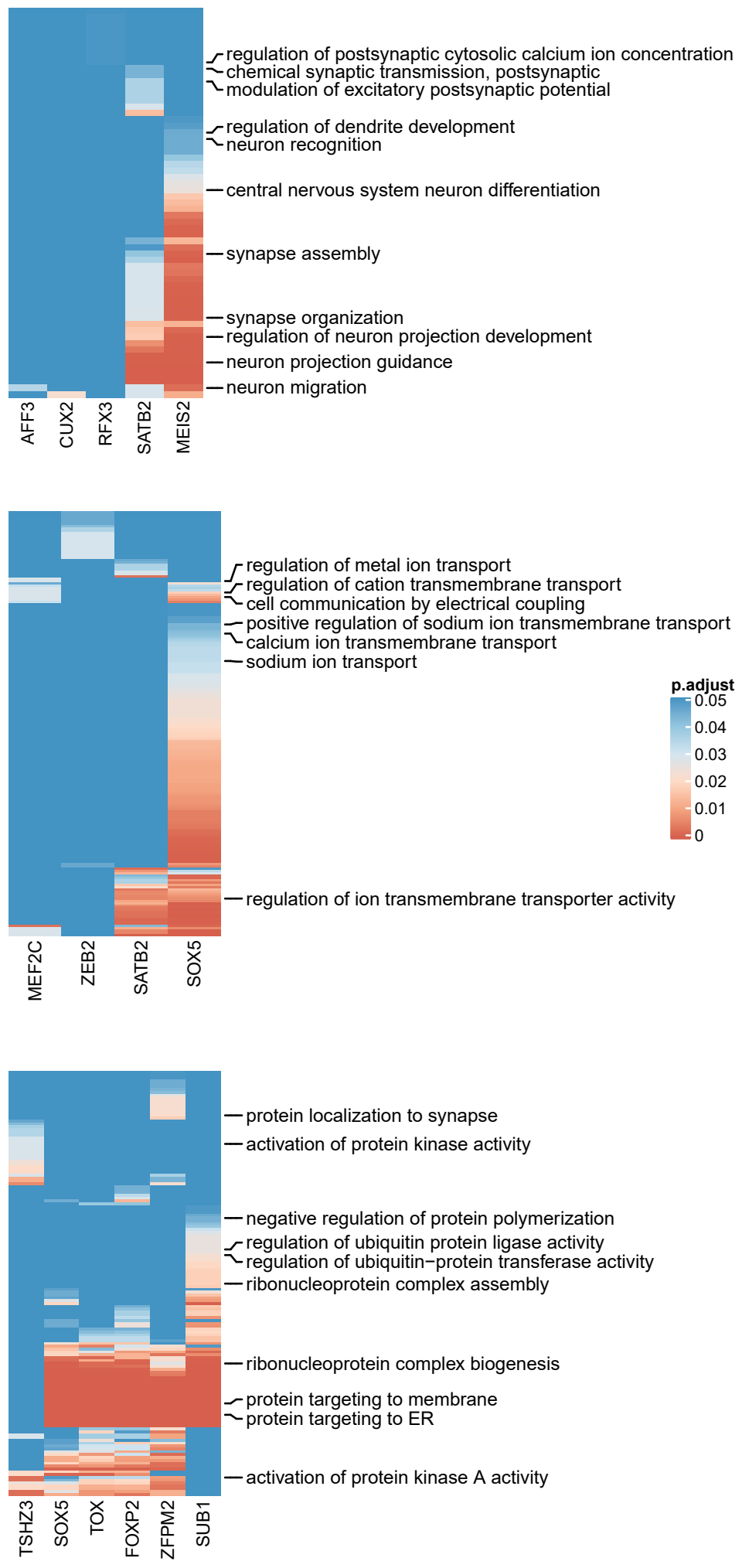